\title{
The effects of industry funding and positive outcomes in the interpretation of clinical trial results: a randomized trial among Dutch psychiatrists
}

\author{
Joeri K. Tijdink ${ }^{1,2^{*}}$, Yvo M. Smulders ${ }^{3}$, Lex M. Bouter ${ }^{1,4}$ and Christiaan H. Vinkers ${ }^{5}$
}

\begin{abstract}
Background: Most studies are inclined to report positive rather than negative or inconclusive results. It is currently unknown how clinicians appraise the results of a randomized clinical trial. For example, how does the study funding source influence the appraisal of an RCT, and do positive findings influence perceived credibility and clinical relevance? This study investigates whether psychiatrists' appraisal of a scientific abstract is influenced by industry funding disclosures and a positive outcome.
\end{abstract}

Methods: Dutch psychiatrists were randomized to evaluate a scientific abstract describing a fictitious RCT for a novel antipsychotic drug. Four different abstracts were created reporting either absence or presence of industry funding disclosure as well as a positive or a negative outcome. Primary outcomes were the perceived credibility and clinical relevance of the study results (10-point Likert scale). Secondary outcomes were the assessment of methodological quality and interest in reading the full article.

Results: Three hundred ninety-five psychiatrists completed the survey (completion rate 45\%). Industry funding disclosure was found not to influence perceived credibility (Mean Difference MD 0.12; $95 \% \mathrm{Cl}-0.28$ to $0.47, \mathrm{p}$ ?) nor interpretation of its clinical relevance (MD $0.14 ; 95 \% \mathrm{Cl}-0.54$ to $0.27, \mathrm{p}$ ?). A negative outcome was perceived as more credible than a positive outcome (MD 0.81 points; $95 \%$ Confidence Interval (Cl) 0.43 to 1.18, p?), but did not affect clinical relevance scores (MD $-0.14 ; 95 \% \mathrm{Cl}-0.54$ to 0.27 ).

Conclusions: In this study, industry funding disclosure was not associated with the perceived credibility nor judgement of clinical relevance of a fictional RCT by psychiatrists. Positive study outcomes were found to be less credible compared to negative outcomes, but industry funding had no significant effects. Psychiatrists may underestimate the influence of funding sources on research results. The fact that physicians indicated negative outcomes to be more credible may point to more awareness of existing publication bias in the scientific literature.

Keywords: Industry funding, Conflict of interest, Positive outcome bias, Credibility, Industry funding disclosure, Psychiatrists, Methodological quality, Perceived credibility, Perceived clinical relevance

\footnotetext{
*Correspondence: j.tijdink@amsterdamumc.nl

'Department of Philosophy, Faculty of Humanities, VU Vrije Universiteit, 1081

HZ Amsterdam, Netherlands

${ }^{2}$ Department of Medical Humanities, Amsterdam UMC, (location VUmc),

Amsterdam, the Netherlands

Full list of author information is available at the end of the article
}

(c) The Author(s). 2019 Open Access This article is distributed under the terms of the Creative Commons Attribution 4.0 International License (http://creativecommons.org/licenses/by/4.0/), which permits unrestricted use, distribution, and reproduction in any medium, provided you give appropriate credit to the original author(s) and the source, provide a link to the Creative Commons license, and indicate if changes were made. The Creative Commons Public Domain Dedication waiver (http://creativecommons.org/publicdomain/zero/1.0/) applies to the data made available in this article, unless otherwise stated. 


\section{Background}

Several factors are known to influence the interpretation of research findings by editors, reviewers, and scientists [1-4]. Of these factors, study outcome and funding disclosure are important [2,5]. The excess of positive results in the scientific literature, most likely due to selective reporting, is widely acknowledged [6, 7]. In addition, it has been firmly established that industry sponsored studies more frequently report positive results when compared to non-sponsored trials $[8,9]$, and that 'negative' industry-sponsored studies remain unpublished [10-13]. However, a limited number of studies have addressed factors that influence physicians in their interpretation of the scientific literature. This topic is important since physicians often fail to recognize the impact of conflicts of interest [14], even though a critical attitude by medical doctors towards industry funding has been found to affect the perceived relevance of a study [15]. In this context, psychiatry is a field of particular interest. Psychiatrists are frequently criticized for their ties to the pharmaceutical industry and the impact of these ties has been intensely debated by top flight journals [16-18]. Pharmaceutical companies have been criticized for the high profits they received from antidepressants and antipsychotic sells. Their sells may have been influenced by substantial publication bias [13]. Furthermore, a controversy has emerged from reanalysis of data from sponsored study 329 that showed serious side effects of antidepressants which were not reported in the initial study [19]. Nevertheless, it is unknown whether these and other factors such as study outcomes and industry funding influence the scientific evaluation of an RCT. If psychiatrists are easily swayed by funding disclosure or positive outcomes, this could have direct impact on their prescribing behavior and influence their clinical decision making. Therefore, we aimed to assess how study outcome and industry funding disclosure influence psychiatrists' perception of credibility and clinical relevance of results of a hypothetical RCT.

\section{Methods}

\section{Abstract development}

Three of the authors of this study extensively discussed the content of the proposed fictitious abstracts. We used the PubMed format (reproduced without the NCBI logo in Additional file 2: SF1-4) to make it appear like an original study. After producing a first draft of the abstracts by three authors, it was sent as a pilot version to 5 other psychiatrists to receive feedback on formulation, design, credibility and face-validity. This feedback resulted in minor modifications and thus the final version of the abstract. Each participant received the survey in Dutch containing an abstract in the English language describing an RCT for a non-existing novel antipsychotic drug 'vinquerine' in a fictitious journal ("the ArXX CXXX PsychXXX"). We chose to display the abstract in English since Dutch psychiatrists are comfortable in reading scientific literature in English. Four different abstracts were created based on reported study outcome (positive (a statistical significant difference compared with olanzapine) vs negative (no statistical differences compared with olanzapine)) and industry funding disclosure (yes/no). The original fake abstracts are included in (Additional file 2: Figures SF1-4). The fictional study compared the effects of the antipsychotic medication vinquerine to those of olanzapine and placebo on psychotic symptoms in patients with a first-episode psychosis. We chose olanzapine because it is an established and often used treatment for psychosis. Severe side effects were recorded in the fictitious abstract since these influence prescription behavior; the presence or absence of side effects often makes significant influence on the choices clinicians make in the treatment of psychiatric patients.

A positive outcome was reported by showing a statistically and clinically significant effect of vinquerine compared to olanzapine (an often applied first line treatment for psychosis) and placebo. In the positive outcome abstract, vinquerine was reported to have very limited side effects.

A negative outcome was reported as vinquerine and olanzapine being equally effective, but vinquerine showing important side effects. Both vinquerine and olanzapine were reported as being superior to placebo.

In the industry funded version, the second author was reported to be a consultant for a fictional pharmaceutical company ('Olevy Pharmaceuticals'). We chose to put the conflict of interest (CoI) under the second author, rather than the first, since it was considered to be more consistent with existing scientific reporting. At the bottom of the fictitious industry funded abstracts, it was clearly stated that 'this study was funded by a research grant from Olevy Pharmaceuticals'.

Identical methodological limitations were deliberately introduced in all versions of the abstract to make it consistent with Pubmed abstracts that, according to the authors, were judged to be average quality. We felt that reporting methods of average quality would make it more likely that the article would be scrutinized for validity. In contrast, a study without noticeable limitations could be received by clinicians as being fabricated or lacking veracity. The limitations of the fictitious abstract included a relatively small sample size $(n=303)$, unclear selection of study participants, non-blinded study design, a relatively short follow-up period (4-weeks), and the exclusion of non-compliant patients in a per-protocol analysis. The abstract-only approach was deliberately chosen since physicians frequently only read the abstract of potentially interesting scientific studies [20]. 
Statement: To prevent a potential confusion, we would like to underline that the abstracts were invented.

\section{Survey sample and randomization}

VanDerHoef\&Partners provided access to Dutch psychiatrists but were not involved in any part of the study (concept, design, analysis, and writing). One thousand five hundred sixty-six Dutch psychiatrists were randomized to receive an e-mail with a link to one of the following four abstracts: 1) negative outcome/industry funding disclosure, 2) negative outcome/no industry funding disclosure, 3) positive outcome/industry funding disclosure, or 4) positive outcome/no industry funding disclosure. The invitational e-mail and subsequent reminders were sent in May and June 2014 and included a brief statement to describe the goal of the study in very general terms ('to determine how psychiatrists evaluate scientific research'). Participants were left unaware of the true design and intention of the study. The e-mail included a link to the online survey and the instructions on how-to opt-out of this study if they so chose. Two reminders were sent within a four week time frame to psychiatrists who did not respond to the initial email. If psychiatrists declined to respond, they were asked to follow an electronic link to a very brief online questionnaire to disclose their reasons for non-participation. Consent to participate was implied by following the link to the survey and completing the survey.

\section{Survey characteristics and outcomes}

Each abstract was accompanied by a short questionnaire with 10 statements to determine the credibility ("How would you rate the credibility of the abstract?"), clinical relevance, methodological rigor (seven statements), and interest in reading the full article using a 10-points Likert scale (1: very poor, 10: extremely good). A control question ("please enter the score 3 for this question") was included to check if participants did not randomly complete the survey. Methodological rigor was assessed with seven items, addressing different methodological characteristics: study design, methodology, statistical analysis, sample size, outcome measures, completeness of reporting and overall study quality [see Additional file 1: Table S1]. An equal-weighted sum score for the 7 items was calculated. To prevent that the order of questions have an undue influence on the answers respondents provided, the primary outcome questions were randomly distributed among the 10 questions.

After completion of the survey, participants' attitudes towards the pharmaceutical industry were assessed. We also asked for financial ties with the pharmaceutical industry. Participants were asked if they had received a representative of a pharmaceutical company or if they had received any industry funding in the past 6 months.
In addition, they were provided with four statements regarding the influence of industry funding on scientific clinical studies. Participants were asked to agree or disagree. At this stage of the survey, participants were not able to alter any of their previous answers.

Participant characteristics were obtained by asking respondents to report their gender, age, professional affiliations (academia, general hospital, or mental health care facility) and whether or not they had obtained a $\mathrm{PhD}$. In addition, they were asked their place of residency, and whether the participant was actively involved in scientific research. For the complete survey with all the questions, see Additional file 3.

\section{Statistical analysis}

Primary outcome measures were the perceived credibility and the judgement regarding clinical relevance of the study results reported in the fictitious abstract. Secondary outcomes were the level of interest in reading the full article, and global assessment of methodological rigor (sum score). All primary and secondary outcomes were scored on a 10-point Likert scale. We checked for distribution patterns of the primary and secondary outcomes and concluded that the distribution allows the use of ANOVA (see Additional file 1: Table S4). The survey software was constructed in a manner that prevented participants from submitting the survey unless all questions were completed. This was done to minimize missing data. First, possible interaction between the industry funding disclosure and study outcome was addressed. For the primary outcomes, the effect of funding disclosure and study outcome were analyzed using $2 \times 2$ ANOVA. Possible confounders and effect modifiers were analyzed, including participants' self-reported attitude towards industry funding, and active relationships with the pharmaceutical industry. As a secondary, exploratory analysis, linear univariate and multivariate regression analyses were used to identify other possible determinants of perceived credibility and clinical relevance, including demographic and job specific factors, and active relations with industry. The Statistical Package for the Social Sciences (SPSS) statistics (Chicago USA 2011, version 20) was used for all statistical analyses.

\section{Results}

\section{Participant characteristics}

A total of 880 psychiatrists opened the invitation by email, to which 580 psychiatrists responded (66\%), and 395 (45\%) completed the full survey. Two respondents were excluded because they failed to correctly answer the control question. Demographic data and characteristics of the participants are presented in Table 1. Attitudes towards industry funding are included in Additional file 1: Tables S2 and S3). 
Table 1 Demographic and professional characteristics of the participants

\begin{tabular}{|c|c|c|c|}
\hline & & $N=395$ & $\%$ \\
\hline Gender & Male (\%) & 214 & 54 \\
\hline Age & Mean (range) & $50(27-72)$ & \\
\hline \multirow[t]{5}{*}{ Professional affiliation } & Academia & 40 & 10 \\
\hline & General Hospital & 37 & 9 \\
\hline & Mental Health Institution & 248 & 63 \\
\hline & Private Practice & 63 & 16 \\
\hline & Other & 38 & 10 \\
\hline \multirow[t]{4}{*}{ Residency background } & Academic Hospital & 158 & 40 \\
\hline & General Hospital & 4 & 1 \\
\hline & Mental Health Institution & 200 & 51 \\
\hline & Other & 32 & 8 \\
\hline \multirow[t]{4}{*}{ Sub-specialty } & Adult psychiatry & 241 & 61 \\
\hline & Child psychiatry & 90 & 23 \\
\hline & Geriatric psychiatry & 25 & 6 \\
\hline & Other & 37 & 9 \\
\hline Received representative of a pharm. Company & Yes & 180 & 46 \\
\hline Received pharmacological funding & Yes & 14 & 4 \\
\hline Scientifically active & Yes & 131 & 33 \\
\hline PhD degree & Yes & 104 & 27 \\
\hline Years employed as psychiatrist (range) & & $14(0-40)$ & \\
\hline
\end{tabular}

\section{Industry funding disclosure and study outcome Interaction analysis}

No significant interaction was found between study outcome in the abstract and mentioning of the industry funding disclosure, both on the primary outcome credibility $(p=0.99)$ and the outcome clinical relevance $(p=0.41)$.

\section{Industry funding disclosure does not affect credibility or relevance assessments}

Industry funding disclosure was not significantly associated with perceived credibility (MD 0.12; 95\% CI -0.28 to 0.47 ) nor clinical relevance (MD $0.14 ; 95 \% \mathrm{CI}-0.54$ to 0.27 ). Likewise, no significant effects were found for industry funding disclosure on the secondary outcomes 'assessment of methodological rigor' (MD 0.22; 95\% CI -1.82 to 2.17 ), and 'interest in reading the full article' (MD 0.14; $95 \% \mathrm{CI}-0.40$ to 0.71 ) (Table 2).

\section{Positive study outcomes are perceived as less credible} A positive study outcome of the fictional RCT was associated with significantly lower scores on credibility (MD $0.81 ; 95 \%$ CI 0.43 to 1.18 ) but not 'clinical relevance'

Table 2 ANOVA analysis of the primary and secondary outcomes of the abstract with or without funding disclosure and with a positive or negative study outcome

\begin{tabular}{|c|c|c|c|c|}
\hline & Funding $(N=206)$ & No Funding $(N=187)$ & Mean Difference (MD) & $95 \% \mathrm{Cl}$ \\
\hline Credibility $^{a}$ & 4.64 & 4.76 & 0.12 & -0.28 to 0.47 \\
\hline Clinical relevance & 5.30 & 5.16 & 0.14 & -0.54 to 0.27 \\
\hline Interest in reading the full article & 4.51 & 4.65 & 0.14 & -0.40 to 0.71 \\
\hline \multirow[t]{2}{*}{ Methodological quality (sum score) } & 38.65 & 38.87 & 0.22 & -1.82 to 2.17 \\
\hline & Negative outcome $(N=200)$ & Positive outcome $(N=193)$ & Mean Difference (MD) & $95 \% \mathrm{Cl}$ \\
\hline Credibility $^{a}$ & 5.10 & 4.29 & 0.81 & 0.43 to 1.18 \\
\hline Clinical relevance $^{a}$ & 5.16 & 5.30 & 0.14 & -0.28 to 0.53 \\
\hline Interest in reading the full article ${ }^{a}$ & 4.31 & 4.85 & 0.54 & 0.09 to 1.12 \\
\hline Methodological quality (sum score) ${ }^{b}$ & 5,63 & 5,44 & 0.19 & -3.31 to 0.68 \\
\hline
\end{tabular}

${ }^{\mathrm{a}}$ 10-point Likertscale score

${ }^{\mathrm{b}}$ Average score on a 10-point Likertscale of the seven individual items regarding methodological quality (see Additional file 1: Table S1) 
(MD 0.14; $95 \%$ CI -0.28 to 0.53 ), compared to a negative study outcome. The secondary outcome 'interest in reading the full article' (MD 0.54, 95\% CI 0.09 to 1.12) was statistically significant. The other outcome 'assessment of methodological rigor' (MD 0.19; 95\% CI - 3.31 to 0.68 ) was not significantly influenced by the reported study outcome (Table 2).

\section{No effect modification by relations with or attitude towards industry funding}

We investigated whether participants' active relations with- and attitude towards- industry were effect modifiers or confounders in the observed relations between study outcome and the primary outcomes. Both for perceived credibility and clinical relevance, no interactions or confounding were found for pharmaceutical industry relations and attitude towards industry funding (data not shown). Nevertheless, psychiatrists were well aware of possible industry effects, with an average score of 8.0 of 10 for the statement that "a pharmaceutical company can influence study results", a score of 7.4 out of 10 for the statement that "funding has an effect on the quality of research", and 6.9 out of 10 for the statement that "funding from a pharmaceutical company has a negative influence on the validity of research results" (see Additional file 1: Table S3).

\section{Professional characteristics that influence perceived credibility and clinical relevance}

In a secondary exploratory analysis, we investigated whether professional characteristics affect perceived credibility and clinical relevance scores in the total study population. Credibility of the RCT was negatively associated with having scientific experience (e.g. having a $\mathrm{PhD}$ or being scientifically active) (MD $-0.6 ; 95 \% \mathrm{CI}-0.99$ to -0.21 ), and positively associated with having recently received a pharmaceutical representative (MD 0.63; 95\% CI 0.25 to 1.01), funding from a pharmaceutical company (MD 1.27; 95\% CI 0.25 to 2.30 ) or being employed in a general mental health institution (MD 0.46; CI 0,06 to 0,85 ). Multivariate analyses of these variables did not affect any of these results (data not shown).

Psychiatrists interpreted clinical relevance to be lower if they had scientific experience ('having a PhD' or 'being scientifically active') (MD $-0.43 ; 95 \%$ CI 0.85 to -0.02 ), were longer active as a psychiatrist (beta $-0.02 ; 95 \%$ CI -0.04 to 0.00 ) and had received residency training in a general mental health institution rather than an academic hospital (MD 0.40; 95\% CI -0.80 to 0.00 ). Multivariate analysis only showed significant associations for scientific experience and residency training in a general non-academic mental health institution (data not shown).

\section{Discussion}

This study demonstrates that psychiatrists have more confidence in the validity of a negative outcome than a positive outcome of a fictitious study assessing a novel antipsychotic drug. In apparent contrast, we did not find a difference in the evaluation of a scientific abstract when participants were displayed an industry funding disclosure with the fictitious abstract. The reader's attitudes towards or relationships with the pharmaceutical industry did not influence this analysis.

The relative distrust of a positive study outcome in our study is in agreement with the recognition that the medical literature suffers from positive outcome bias $[6,7]$, particularly in drug studies [21, 22]. Positive outcome bias has also been convincingly demonstrated in psychiatric literature, for example on the effectiveness of antidepressants [4, 23] and antipsychotics [24]. Presumably, respondents were aware of this bias, generating hesitation to accept results that may be considered 'too good to be true'. The fact that positive outcomes are more critically appraised by psychiatrists is consistent with another study that highlights the influence of positive outcomes in paper acceptance rates in peer review [2]. These results are not fully comparable as peer reviewers have other criteria to assess a scientific paper than clinicians.

Although the effects of industry funding on study outcomes has been well-established both in the general medical literature [5] and in the psychiatric literature [11], no effect was found for funding disclosure on the credibility or perceived relevance of, in particular the positive outcome version of the abstract. Moreover, participants' attitudes towards pharmaceutical industry funding did not influence participants' appraisal of the fictitious abstract. (see Additional file 1: Table S3). Participants with overall high scores on statements that pharmaceutical companies can unduly influence study results were no more likely to question the validity of the fictitious abstract, even though the abstract clearly disclosed the funding. We cannot infer why these attitudes did not affect the results of our study. Apparently, psychiatrists did not automatically connect their perception of funding effects to the actual interpretation of the abstract we sent them. It may well be that respondents account for the mentioned disclosures, and may think that their knowledge will not influence the perception of research results.

Disclosure of industry funding in itself does not make study results more or less valid [25]. Future research should investigate whether funding disclosures on scientific abstracts are warranted. One might argue that reporting industry disclosures could unjustifiable influence the perceived credibility of research results simply 
because it was funded by or carried out by the pharmaceutical industry.

The recent media attention on study 329 regarding antidepressant drugs reinforces the need for individual patient data and original study protocols to increase the validity of scientific results as registration of clinical trials is insufficient [19].

Participants were a random sample from \pm 3500 Dutch psychiatrists. Nevertheless, around $27 \%$ of the participants had a $\mathrm{PhD}$ degree, which is higher than the average among community psychiatrists. Probably, psychiatrists with a $\mathrm{PhD}$ are more likely to participate in our survey, as they would feel more competent to assess a scientific abstract. Moreover, in light of the response rate of $45 \%$, we checked whether the study results are generalizable to the total population of Dutch psychiatrists. With $54 \%$ of the survey sample being male compared to $59 \%$ in another recent study of Dutch psychiatrists, [26], there is no reason to assume that the results are not generalizable.

Secondary analyses showed that credibility scores were higher in psychiatrists with active relations with pharmaceutical companies and this was independent of industry funding disclosure. Credibility scores of the fictitious abstract were lower for the psychiatrists with scientific research experience. These results suggest that participants' relationships with the pharmaceutical industry can influence study appraisal in a more positive or less critical manner.

To our knowledge, this is the first randomized study to investigate whether industry funding disclosure and study outcomes can influence the opinion of psychiatrists on the credibility and relevance of clinical research. In contrast, a somewhat smaller, previous study among 269 internists suggested that industry funding disclosure resulted in lower scores on methodological rigor and less confidence in the results [15]. The study design with different methodological approaches and the inclusion of three hypothetical drugs may have played a role in the discrepancy between these two studies. Additionally, it may be possible that internists are more critical towards pharmaceutical industry funded studies compared to psychiatrists.

The results of this study should be cautiously interpreted in light of several limitations. First, internet-based questionnaires can suffer from a selective response bias, predominantly attracting participants who feel capable of assessing a scientific abstract. In our study, the response rate of $45 \%$ was relatively high compared to other internet-based surveys for physicians [27, 28]. To calculate the response rate, we used the psychiatrists who opened the email as the denominator. A stricter calculation of the response rate would use all invitees $(n=$ 1566) - whether they opened the email or not - in our response rate determination. This would have resulted in a response rate of $25 \%$ (395 out of 1566 invitations). We did not survey reasons for non-response. Most likely, psychiatrists lack time and energy to engage in online surveys. Non-response could also be due to the topic of the survey. Possibly, some psychiatrists might have been reluctant to participate as they could feel not competent enough to judge a scientific abstract on its quality.

Second, a 19\% difference (Cohen's d 0.43) in perceived credibility score and the $13 \%$ difference (Cohen's d 0.19) in interest in reading the full article between the two groups is large enough to allow firm conclusions. Third, we did not find a significant difference between funding disclosure and perceived credibility. Although it may be enticing to conclude that there is no relation between the two variables, we cannot draw this conclusion from our data as no evidence of an association is not evidence of no association. Fourth, it might be that the participants perceived the positive study as invalid because olanzapine is a well-established reference antipsychotic drug. Furthermore, they might have perceived the negative study as more realistic in terms of symptom reduction. Fifth, the side effect profile was different in the positive outcome abstract compared to the negative outcome abstract. This was done intentionally to assure that the positive outcome abstract would be perceived as a positive study. However, this may have had a collateral effect on the perceived credibility. Reporting severe side effects might improve the intuitive credibility of an abstract as psychiatrists frequently discuss side effects with patients and are focused on side effects in their treatments.

Finally, the question remains if the responders did notice the presence of the disclosure of industry funding. Some respondents may not have noticed the disclosure of industry funding in the scientific abstract, even though all seven psychiatrists who pretested the fictitious abstract noticed it. Participants may also have assumed that the study on a novel antipsychotic would automatically be funded by industry even without an explicit disclosure statement.

\section{Conclusion}

In this randomized study among psychiatrists, the effect of industry funding disclosure was not associated with the reliability and relevance of the results of a randomized clinical trial for a fictitious antipsychotic drug. In contrast, psychiatrists more critically interpreted the abstract of the RCT when a positive outcome was reported. Our results are timely in light of the recent discussion on the effects of industry-physician relations in several leading medical journals $[16,17,29]$. There is a striking discrepancy between psychiatrists' attitudes towards the 
pharmaceutical industry and the effects that funding disclosure has on their perceived credibility and judgement of clinical relevance of the RCT.

Nevertheless, the credibility of the RCT was lower in psychiatrists with more scientific experience and higher when psychiatrists received a pharmaceutical representative or received funding from a pharmaceutical company. Future research could investigate whether explicit funding disclosures on scientific abstracts in databases such as warranted.

\section{Additional files}

Additional file 1: Table S1. Perceived methodological rigor Average scores of individuals statements regarding the methodological rigor of the abstract. Table S2. Attitudes towards pharmaceutical funding related to credibility and clinical relevance Univariate regression analysis examining whether attitudes towards industry funding affects the perceived credibility and clinical relevance. Regression coefficients and 95\% confidence intervals ( $\mathrm{Cls}$ ) are shown. Table S3. Attitude towards pharmaceutical funding Mean scores of four statements regarding the attitude of participants towards pharmaceutical companies and their involvement in research. Table S4. Distribution of primary and secondary outcomes. (DOC $52 \mathrm{~kb}$ )

Additional file 2: SF 1. Pubmed style fake abstract with positive outcome and funding disclosure. SF 2 . Pubmed style fake abstract with positive outcome and no funding disclosure. SF $\mathbf{3}$. Pubmed style fake abstract with negative outcome and funding disclosure SF 4. Pubmed style fake abstract with negative outcome and no funding disclosure. (PDF $893 \mathrm{~kb}$ )

Additional file 3. Survey_Questions. (The translation of the survey questions). (DOC $27 \mathrm{~kb}$ )

\section{Abbreviations}

ANOVA: Analysis of Variance; Cl: Confidence Interval; Col: Conflict of Interest; MD: Mean Difference; NCBI: National Center for Biotechnology Information; RCT: Randomized Controlled Trial; SPSS: Statistical Package for the Social Sciences

\section{Acknowledgements}

We would like to thank VanDerHoef \& Partners (Locum tenens company) for providing email addresses of the participating psychiatrists. We would like to thank Dr. James McKinnon, psychiatrist for his critical review of the manuscript in English.

\section{Authors'contributions}

JT, YS, LB and CV all had a substantial contributions to the conception or design of the work; or the acquisition, analysis, or interpretation of data for the work; and JT, YS, LB and CV drafted the work or revising it critically for important intellectual content; and JT, YS, LB and CV gave final approval of the version to be published; and JT, YS, LB and CV all agreed to be accountable for all aspects of the work in ensuring that questions related to the accuracy or integrity of any part of the work are appropriately investigated and resolved. All authors read and approved the final manuscript.

\section{Funding}

There is no funding to declare.

\section{Availability of data and materials}

An anonymized version of the dataset used is available from the corresponding author on reasonable request.

\section{Ethics approval and consent to participate}

The ethical review board of the VU Medical Center considered the research project and decided that formal ethical approval was waived for this study.
Consent to participate was implied by following the link to the survey and completing the survey.

\section{Consent for publication}

Not applicable.

\section{Competing interests}

The authors declare that they have no competing interests.

\section{Author details}

${ }^{1}$ Department of Philosophy, Faculty of Humanities, VU Vrije Universiteit, 1081 HZ Amsterdam, Netherlands. ${ }^{2}$ Department of Medical Humanities, Amsterdam UMC, (location VUmc), Amsterdam, the Netherlands. ${ }^{3}$ Department of Internal Medicine, Amsterdam UMC, (location VUmc), 1081 $\mathrm{HZ}$ Amsterdam, the Netherlands. ${ }^{4}$ Department of Epidemiology \& Biostatistics, Amsterdam UMC (location VUmc), $1081 \mathrm{HZ}$ Amsterdam, the Netherlands. ${ }^{5}$ Department of Psychiatry, and Department of Anatomy and Neurosciences, Amsterdam UMC (location VUmc), Amsterdam, the Netherlands.

Received: 29 December 2017 Accepted: 3 September 2019

Published online: 18 September 2019

\section{References}

1. Angell M. Industry-sponsored clinical research: a broken system. JAMA. 2008:300:1069-71.

2. Emerson GB, Warme WJ, Wolf FM, Heckman JD, Brand RA, Leopold SS Testing for the presence of positive-outcome bias in peer review: a randomized controlled trial. Arch Intern Med. 2010;170:1934-9.

3. Lundh A, Sismondo S, Lexchin J, Busuioc OA, Bero L. Industry sponsorship and research outcome. Cochrane Database Syst Rev. 2012;(12):MR000033.

4. Turner EH, Matthews AM, Linardatos E, Tell RA, Rosenthal R. Selective publication of antidepressant trials and its influence on apparent efficacy. N Engl J Med. 2008;358:252-60

5. Lexchin J, Bero LA, Djulbegovic B, Clark O. Pharmaceutical industry sponsorship and research outcome and quality: systematic review. BMJ. 2003:326:1167-70.

6. Chan AW, Hrobjartsson A, Haahr MT, Gotzsche PC, Altman DG. Empirical evidence for selective reporting of outcomes in randomized trials: comparison of protocols to published articles. JAMA. 2004;291:2457-65.

7. Dwan K, Gamble C, Williamson PR, Kirkham JJ. Systematic review of the empirical evidence of study publication bias and outcome reporting bias an updated review. PLoS One. 2013;8:e66844.

8. Bhandari M, Busse JW, Jackowski D, Montori VM, Schunemann $H$, Sprague $\mathrm{S}$, et al. Association between industry funding and statistically significant pro-industry findings in medical and surgical randomized trials. CMAJ. 2004;170:477-80.

9. Bourgeois FT, Murthy S, Mandl KD. Outcome reporting among drug trials registered in ClinicalTrials.gov. Ann Intern Med 2010;153:158-66.

10. Hopewell S, Loudon K, Clarke MJ, Oxman AD, Dickersin K. Publication bias in clinical trials due to statistical significance or direction of trial results. Cochrane Database Syst Rev. 2009:1:MR000006.

11. Perlis RH, Perlis CS, Wu Y, Hwang C, Joseph M, Nierenberg AA. Industry sponsorship and financial conflict of interest in the reporting of clinical trials in psychiatry. Am J Psychiatry. 2005;162:1957-60.

12. Rising K, Bacchetti P, Bero L. Reporting bias in drug trials submitted to the Food and Drug Administration: review of publication and presentation. PLoS Med. 2008;5:e217.

13. Turner EH. Publication bias, with a focus on psychiatry: causes and solutions. CNS Drugs. 2013;27:457-68.

14. Silverman GK, Loewenstein GF, Anderson BL, Ubel PA, Zinberg S, Schulkin J. Failure to discount for conflict of interest when evaluating medical literature: a randomised trial of physicians. J Med Ethics. 2010:36:265-70.

15. Kesselheim AS, Robertson CT, Myers JA, Rose SL, Gillet V, Ross KM, et al. A randomized study of how physicians interpret research funding disclosures. N Engl J Med. 2012;367:1119-27.

16. Godlee F. Conflict of interest: forward not backward. BMJ. 2015;350:h3176

17. Horton R. Offline: the BMJ vs NEJM--lessons for us all. The Lancet; 2015: Elsevier; 2015. p. 2238

18. Appelbaum P, Arboleda-florez JU, Javed A, Soldatos C, Tyano S. WPA recommendations for relationships of psychiatrists, health care 
organizations working in the psychiatric field and psychiatric associations with the pharmaceutical industry. World Psychiatry. 2011;10(2):155-9.

19. Le Noury J, Nardo JM, Healy D, Jureidini J, Raven M, Tufanaru C, et al. Restoring study 329: efficacy and harms of paroxetine and imipramine in treatment of major depression in adolescence. BMJ. 2015;351:h4320.

20. Saint S, Christakis DA, Saha S, Elmore JG, Welsh DE, Baker P, et al. Journal reading habits of internists. J Gen Intern Med. 2000;15:881-4.

21. Page MJ, McKenzie JE, Kirkham J, Dwan K, Kramer S, Green S, et al. Bias due to selective inclusion and reporting of outcomes and analyses in systematic reviews of randomised trials of healthcare interventions. Cochrane Database Syst Rev. 2014;(10):MR000035.

22. Saini P, Loke YK, Gamble C, Altman DG, Williamson PR, Kirkham JJ. Selective reporting bias of harm outcomes within studies: findings from a cohort of systematic reviews. BMJ. 2014;349:g6501.

23. Roest AM, dJ P. Williams CD, de Vries YA, Schoevers RA, Turner EH. Reporting Bias in clinical trials investigating the efficacy of secondgeneration antidepressants in the treatment of anxiety disorders: a report of 2 meta-analyses. JAMA Psychiatry. 2015;72:500-10.

24. Turner EH, Knoepflmacher D, Shapley L. Publication bias in antipsychotic trials: an analysis of efficacy comparing the published literature to the US Food and Drug Administration database. PLoS Med. 2012;9:e1001189.

25. Loewenstein G, Sah S, Cain DM. The unintended consequences of conflict of interest disclosure. JAMA. 2012;307(7):669-70.

26. Koerselman GF, Smit JH, van Tilburg W. A study of job satisfaction among Dutch psychiatrists. Tijdschrift voor psychiatrie. 2011;53(7):393-403.

27. Asch DA, Jedrziewski MK, Christakis NA. Response rates to mail surveys published in medical journals. J Clin Epidemiol. 1997;50:1129-36.

28. Cook C, Heath F, Thompson RL. A meta-analysis of response rates in webor internet-based surveys. Educ Psychol Meas. 2000;60:821-36.

29. Rosenbaum L. Conflicts of interest: part 1: reconnecting the dots-reinterpreting industry-physician relations. N Engl J Med. 2015;372:1860-4.

\section{Publisher's Note}

Springer Nature remains neutral with regard to jurisdictional claims in published maps and institutional affiliations.

Ready to submit your research? Choose BMC and benefit from:

- fast, convenient online submission

- thorough peer review by experienced researchers in your field

- rapid publication on acceptance

- support for research data, including large and complex data types

- gold Open Access which fosters wider collaboration and increased citations

- maximum visibility for your research: over $100 \mathrm{M}$ website views per year

At $\mathrm{BMC}$, research is always in progress.

Learn more biomedcentral.com/submissions 